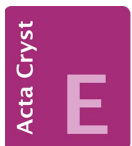

CRYSTALLOGRAPHIC COMMUNICATIONS

ISSN 2056-9890

Received 5 December 2014

Accepted 7 January 2015

Edited by M. Weil, Vienna University of Technology, Austria

Keywords: crystal structure; transition metal phosphates; hydrothermal synthesis;

$\mathrm{Fe}_{3}\left(\mathrm{PO}_{4}\right)_{2} \cdot \mathrm{H}_{2} \mathrm{O}$ structure type

CCDC reference: 1042563

Supporting information: this article has

supporting information at journals.iucr.org/e

\section{Crystal structure of dimanganese(II) zinc bis[orthophosphate(V)] monohydrate}

\author{
Ghaleb Alhakmi,* Abderrazzak Assani, Mohamed Saadi and Lahcen El Ammari \\ Laboratoire de Chimie du Solide Appliquée, Faculté des Sciences, Université Mohammed V, Avenue Ibn Battouta, BP \\ 1014, Rabat, Morocco. *Correspondence e-mail: g_alhakmi@yahoo.fr
}

The title compound, $\mathrm{Mn}_{2} \mathrm{Zn}\left(\mathrm{PO}_{4}\right)_{2} \cdot \mathrm{H}_{2} \mathrm{O}$, was obtained under hydrothermal conditions. The structure is isotypic with other transition metal phosphates of the type $M_{3-x} M_{x}{ }_{x}\left(\mathrm{PO}_{4}\right)_{2} \cdot \mathrm{H}_{2} \mathrm{O}$, but shows no statistical disorder of the three metallic sites. The principal building units are distorted $\left[\mathrm{MnO}_{6}\right]$ and $\left[\mathrm{MnO}_{5}\left(\mathrm{H}_{2} \mathrm{O}\right)\right]$ octahedra, a distorted $\left[\mathrm{ZnO}_{5}\right]$ square pyramid and two regular $\mathrm{PO}_{4}$ tetrahedra. The connection of the polyhedra leads to a framework structure. Two types of layers parallel to $(\overline{101})$ can be distinguished in this framework. One layer contains $\left[\mathrm{Zn}_{2} \mathrm{O}_{8}\right]$ dimers linked to $\mathrm{PO}_{4}$ tetrahedra via common edges. The other layer is more corrugated and contains $\left[\mathrm{Mn}_{2} \mathrm{O}_{8}\left(\mathrm{H}_{2} \mathrm{O}\right)_{2}\right]$ dimers and $\left[\mathrm{MnO}_{6}\right]$ octahedra linked together by common edges. The $\mathrm{PO}_{4}$ tetrahedra link the two types of layers into a framework structure with channels parallel to [101]. The $\mathrm{H}$ atoms of the water molecules point into the channels and form $\mathrm{O}-\mathrm{H} \cdots \mathrm{O}$ hydrogen bonds (one of which is bifurcated) with framework $\mathrm{O}$ atoms across the channels.

\section{Chemical context}

The great structural diversity of metal-based phosphates, associated with their physical properties makes this family of compounds interesting as potential functional materials, e.g. as catalysts (Viter \& Nagornyi, 2009; Weng et al., 2009) or ionexchangers (Jignasa et al., 2006). Among the wide variety of metal phosphates, one of our interests is focused on mixed metallic orthophosphates of general formula $M_{3-x} M^{\prime}{ }_{x}\left(\mathrm{PO}_{4}\right)_{2} \cdot \mathrm{H}_{2} \mathrm{O}$. The present communication reports the hydrothermal synthesis and structural characterization of a new member of this family, $\mathrm{Mn}_{2} \mathrm{Zn}\left(\mathrm{PO}_{4}\right)_{2} \cdot \mathrm{H}_{2} \mathrm{O}$.

\section{Structural commentary}

The structure of the title compound is built up from four different types of building units: $\left[\mathrm{MnO}_{6}\right]$ and $\left[\mathrm{MnO}_{5}\left(\mathrm{H}_{2} \mathrm{O}\right)\right]$ octahedra, $\left[\mathrm{ZnO}_{5}\right]$ square pyramids and $\mathrm{PO}_{4}$ tetrahedra, as shown in Fig. 1. Whereas the $\left[\mathrm{MnO}_{6}\right]$ octahedron is more or less regular with $\mathrm{Mn}-\mathrm{O}$ distances in the range 2.1254 (13) to 2.2590 (13) $\AA$, the $\left[\mathrm{MnO}_{5}\left(\mathrm{H}_{2} \mathrm{O}\right)\right]$ octahedron is significantly distorted with five equal $\mathrm{Mn}-\mathrm{O}$ distances in the range 2.1191 (13) to 2.1556 (16) and one considerably longer $\mathrm{Mn}-\mathrm{O}$ distance to the water ligand of 2.5163 (15) $\AA$; the $\mathrm{ZnO}_{5}$ square pyramid is also distorted with four shorter $\mathrm{Zn}-\mathrm{O}$ distances between 1.9546 (13) and 2.0347 (12) $\AA$ and one longer $\mathrm{Zn}-\mathrm{O}$ distance, likewise to the water O atom [2.3093 (14) $\AA$ ]; the two $\mathrm{PO}_{4}$ tetrahedra are rather regular $[\mathrm{P}-\mathrm{O}$ distances between 1.5322 (13) and $1.5570(13) \AA$; $\mathrm{O}-\mathrm{P}-\mathrm{O}$ angles between $102.92(7)$ and $\left.111.62(8)^{\circ}\right]$. These polyhedra are arranged in such a way as to build up two types of layers parallel to (101). 


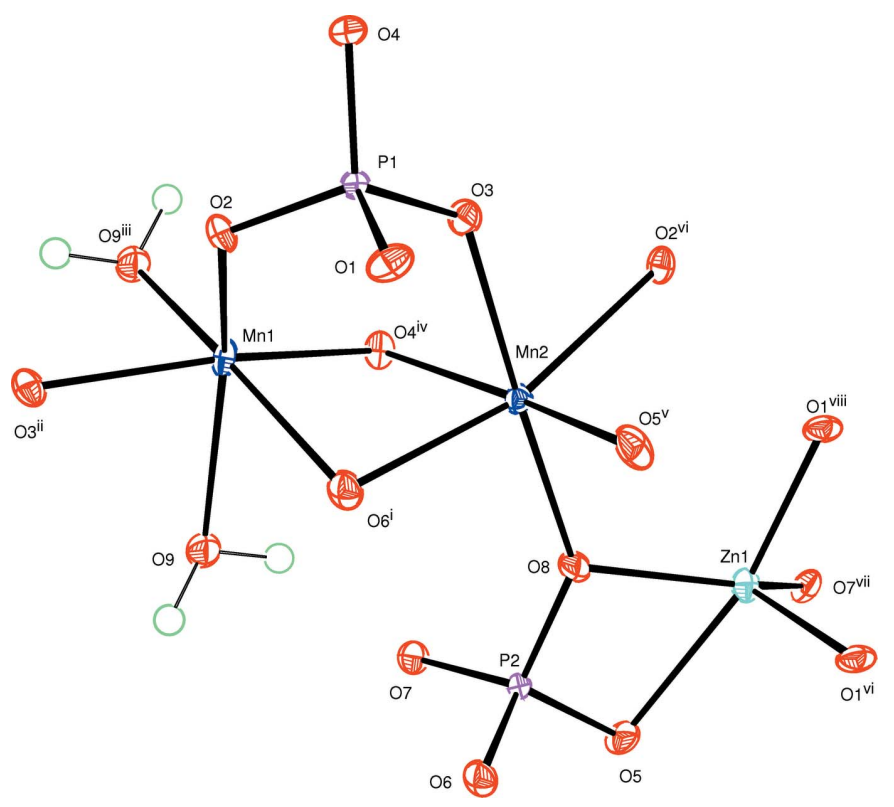

Figure 1

The principal building units in the structure of $\mathrm{Mn}_{2} \mathrm{Zn}\left(\mathrm{PO}_{4}\right)_{2} \cdot \mathrm{H}_{2} \mathrm{O}$. Displacement ellipsoids are drawn at the $50 \%$ probability level. Hydrogen bonds are indicated by dashed lines. [Symmetry codes: (i) $-x+1,-y+1,-z+1$; (ii) $x+\frac{1}{2},-y+\frac{1}{2}, z+\frac{1}{2}$; (iii) $-x+2,-y+1,-z+1$; (iv) $-x+\frac{3}{2}, y+\frac{1}{2},-z+\frac{1}{2}$; (v) $-x+\frac{1}{2}, y-\frac{1}{2},-z+\frac{1}{2}$; (vi) $x-\frac{1}{2},-y+\frac{1}{2}, z-\frac{1}{2}$; (vii) $x-\frac{1}{2},-y+\frac{3}{2}, z-\frac{1}{2}$; (viii) $-x+\frac{1}{2}, y+\frac{1}{2},-z+\frac{1}{2}$.]

One layer contains two $\left[\mathrm{ZnO}_{5}\right]$ polyhedra linked together by edge-sharing into a $\left[\mathrm{Zn}_{2} \mathrm{O}_{8}\right]$ dimer that in turn is linked to $\mathrm{PO}_{4}$ tetrahedra. The other layer contains dimers of the type $\left[\mathrm{Mn}_{2} \mathrm{O}_{8}\left(\mathrm{H}_{2} \mathrm{O}\right)_{2}\right]$ (also formed by edge-sharing of two $\left[\mathrm{MnO}_{5}\left(\mathrm{H}_{2} \mathrm{O}\right)\right]$ octahedra), connecting $\left[\mathrm{MnO}_{6}\right]$ octahedra and $\mathrm{PO}_{4}$ tetrahedra through common vertices. The two types of layers are linked by common edges and vertices into a

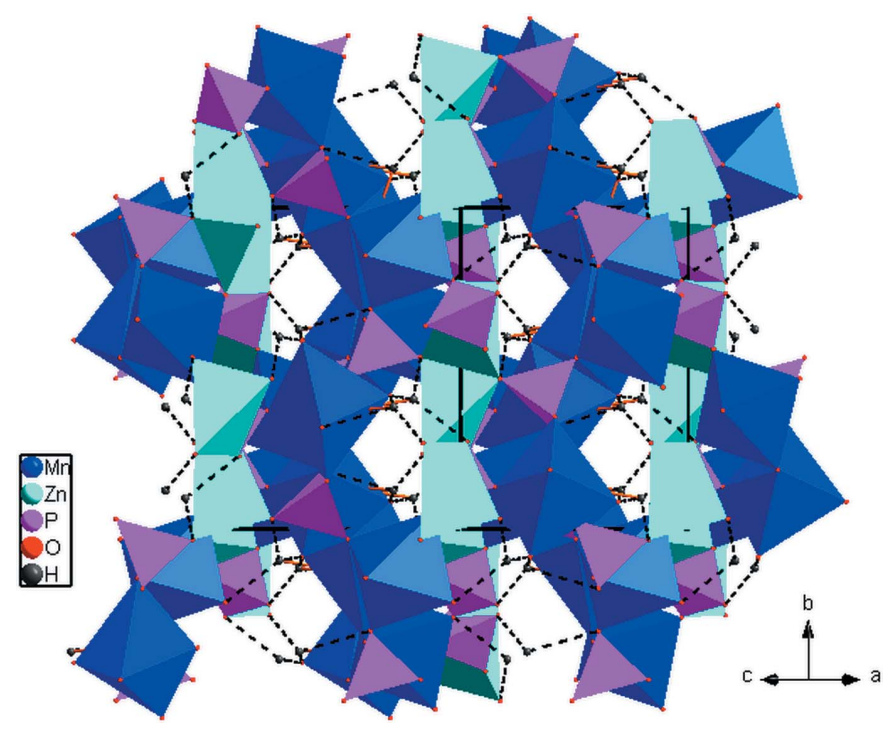

Figure 2

Polyhedral representation of $\mathrm{Mn}_{2} \mathrm{Zn}\left(\mathrm{PO}_{4}\right)_{2} \cdot \mathrm{H}_{2} \mathrm{O}$ showing channels extending parallel to [101]. Hydrogen bonds are shown as dashed lines.
Table 1

Hydrogen-bond geometry $\left(\AA,^{\circ}\right)$.

\begin{tabular}{lllll}
\hline$D-\mathrm{H} \cdots A$ & $D-\mathrm{H}$ & $\mathrm{H} \cdots A$ & $D \cdots A$ & $D-\mathrm{H} \cdots A$ \\
\hline O9-H1 $\cdots \mathrm{O} 7$ & 0.89 & 1.97 & $2.7866(19)$ & 151 \\
O9-H2 $^{\mathrm{i}}$ & 0.91 & 2.16 & $2.8687(19)$ & 134 \\
O9-H2 $^{\text {ii }}$ O $^{\text {ii }}$ & 0.91 & 2.48 & $3.0494(19)$ & 120 \\
\hline
\end{tabular}

Symmetry codes: (i) $x+\frac{1}{2},-y+\frac{3}{2}, z+\frac{1}{2}$; (ii) $x+\frac{1}{2},-y+\frac{1}{2}, z+\frac{1}{2}$.

framework structure with channels parallel to [101]. The water molecules of the $\left[\mathrm{MnO}_{5}\left(\mathrm{H}_{2} \mathrm{O}\right)\right]$ octahedra protrude into these channels and develop hydrogen bonds (one bifurcated) of medium-to-weak strength to framework $\mathrm{O}$ atoms across the channels (Fig. 2; Table 1).

The title compound adopts the $\mathrm{Fe}_{3}\left(\mathrm{PO}_{4}\right)_{2} \cdot \mathrm{H}_{2} \mathrm{O}$ structure type (Moore \& Araki, 1975) and is isotypic with various structures of general formula $M_{3-x} M^{\prime}{ }_{x}\left(\mathrm{PO}_{4}\right)_{2} \cdot \mathrm{H}_{2} \mathrm{O}: \mathrm{CuMn}_{2^{-}}$ $\left(\mathrm{PO}_{4}\right)_{2} \cdot \mathrm{H}_{2} \mathrm{O}$ (Liao et al., 1995); $\mathrm{Co}_{2.59} \mathrm{Zn}_{0.41}\left(\mathrm{PO}_{4}\right)_{2} \cdot \mathrm{H}_{2} \mathrm{O}$ (Sørensen et al., 2005); $\mathrm{Co}_{2.39} \mathrm{Cu}_{0.61}\left(\mathrm{PO}_{4}\right)_{2} \cdot \mathrm{H}_{2} \mathrm{O}$ (Assani et al., 2010); $\mathrm{Mg}_{1.65} \mathrm{Cu}_{1.35}\left(\mathrm{PO}_{4}\right)_{2} \cdot \mathrm{H}_{2} \mathrm{O}$ (Khmiyas et al. 2015).

\section{Synthesis and crystallization}

Crystals of $\mathrm{Mn}_{2} \mathrm{Zn}\left(\mathrm{PO}_{4}\right)_{2} \cdot \mathrm{H}_{2} \mathrm{O}$ were obtained by hydrothermal treatment of zinc oxide $(0.0406 \mathrm{~g})$, metallic manganese $(0.0824 \mathrm{~g})$, phosphoric acid $(0.1 \mathrm{ml})$ and $12.5 \mathrm{ml}$ of distilled water, in a proportion corresponding to the molar ratio $\mathrm{Zn}: \mathrm{Mn}: \mathrm{P}=1: 3: 3$. The hydrothermal reaction was

Table 2

Experimental details.

\begin{tabular}{|c|c|}
\hline \multicolumn{2}{|l|}{ Crystal data } \\
\hline Chemical formula & $\mathrm{Mn}_{2} \mathrm{Zn}\left(\mathrm{PO}_{4}\right)_{2} \cdot \mathrm{H}_{2} \mathrm{O}$ \\
\hline$M_{\mathrm{r}}$ & 383.21 \\
\hline Crystal system, space group & Monoclinic, $P 2_{1} / n$ \\
\hline Temperature $(\mathrm{K})$ & 296 \\
\hline$a, b, c(\AA)$ & $8.1784(2), 10.1741$ (2), 9.0896 (2) \\
\hline$\beta\left({ }^{\circ}\right)$ & $114.142(1)$ \\
\hline$V\left(\AA^{3}\right)$ & $690.17(3)$ \\
\hline$Z$ & 4 \\
\hline Radiation type & Mo $K \alpha$ \\
\hline$\mu\left(\mathrm{mm}^{-1}\right)$ & 7.54 \\
\hline Crystal size (mm) & $0.32 \times 0.27 \times 0.19$ \\
\hline \multicolumn{2}{|l|}{ Data collection } \\
\hline Diffractometer & Bruker X8 APEX \\
\hline Absorption correction & $\begin{array}{l}\text { Multi-scan (SADABS; Bruker, } \\
\text { 2009) }\end{array}$ \\
\hline$T_{\min }, T_{\max }$ & $0.574,0.748$ \\
\hline $\begin{array}{l}\text { No. of measured, independent and } \\
\text { observed }[I>2 \sigma(I)] \text { reflections }\end{array}$ & $11327,2407,2305$ \\
\hline$R_{\text {int }}$ & 0.023 \\
\hline$(\sin \theta / \lambda)_{\max }\left(\AA^{-1}\right)$ & 0.746 \\
\hline \multicolumn{2}{|l|}{ Refinement } \\
\hline$R\left[F^{2}>2 \sigma\left(F^{2}\right)\right], w R\left(F^{2}\right), S$ & $0.019,0.052,1.11$ \\
\hline No. of reflections & 2407 \\
\hline No. of parameters & 127 \\
\hline $\mathrm{H}$-atom treatment & H-atom parameters constrained \\
\hline$\Delta \rho_{\max }, \Delta \rho_{\min }\left(\mathrm{e} \AA^{-3}\right)$ & $0.94,-0.84$ \\
\hline
\end{tabular}

Computer programs: APEX2 and SAINT (Bruker, 2009), SHELXS97 and SHELXL97 (Sheldrick, 2008), ORTEP-3 for Windows (Farrugia, 2012), DIAMOND (Brandenburg, 2006) and publCIF (Westrip, 2010). 
conducted in a $23 \mathrm{ml}$ Teflon-lined autoclave under autogenous pressure at $493 \mathrm{~K}$ for five days. After being filtered, washed with deionized water and dried in air, the reaction product consisted of two types of crystals, the first as off-white parallelepipeds corresponding to $\mathrm{Mn}_{7}\left(\mathrm{PO}_{4}\right)_{2}\left(\mathrm{HPO}_{4}\right)_{4}$ (Riou et al., 1987) and the second as colourless parallelepipeds corresponding to the title compound.

\section{Refinement}

Crystal data, data collection and structure refinement details are summarized in Table 2. The O-bound $\mathrm{H}$ atoms were initially located in a difference map. In the last refinement cycle the distances were fixed at 0.89 and $0.91 \AA$, respectively, and the $\mathrm{H}$ atoms refined in the riding-model approximation with $U_{\text {iso }}(\mathrm{H})$ set to $1.5 U_{\text {eq }}(\mathrm{O})$. The highest peak and the deepest hole in the final Fourier map are at $0.32 \AA$ and $0.30 \AA$, respectively, from $\mathrm{Mn} 1$ and $\mathrm{Zn} 1$.

\section{Acknowledgements}

The authors thank the Unit of Support for Technical and Scientific Research (UATRS, CNRST) for the X-ray measurements and Mohammed V University, Rabat, Morocco, for financial support.

\section{References}

Assani, A., Saadi, M. \& El Ammari, L. (2010). Acta Cryst. E66, i44. Brandenburg, K. (2006). DIAMOND. Crystal Impact GbR, Bonn, Germany.

Bruker (2009). APEX2, SAINT and SADABS. Bruker AXS Inc., Madison, Wisconsin, USA.

Farrugia, L. J. (2012). J. Appl. Cryst. 45, 849-854.

Jignasa, A., Rakesh, T. \& Uma, C. (2006). J. Chem. Sci. 118, 185-189.

Khmiyas, J., Assani, A., Saadi, M. \& El Ammari, L. (2015). Acta Cryst. E71, 55-57.

Liao, J. H., Leroux, F., Guyomard, D., Piffard, Y. \& Tournoux, M. (1995). Eur. J. Solid State Inorg. Chem. 32, 403-414.

Moore, P. B. \& Araki, T. (1975). Am. Mineral. 60, 454-459.

Riou, A., Cudennec, Y. \& Gerault, Y. (1987). Acta Cryst. C43, 821823.

Sheldrick, G. M. (2008). Acta Cryst. A64, 112-122.

Sørensen, M. B., Hazell, R. G., Bentien, A., Bond, A. D. \& Jensen, T. R. (2005). Dalton Trans. pp. 598-606.

Viter, V. N. \& Nagornyi, P. G. (2009). Russ. J. Appl. Chem. 82, 935939.

Weng, W., Lin, Z., Dummer, N. F., Bartley, J. K., Hutchings, G. J. \& Kiely, C. J. (2009). Microsc. Microanal. 15, 1438-1439.

Westrip, S. P. (2010). J. Appl. Cryst. 43, 920-925. 


\section{supporting information}

Acta Cryst. (2015). E71, 154-156 [doi:10.1107/S2056989015000341]

\section{Crystal structure of dimanganese(II) zinc bis[orthophosphate(V)] monohydrate}

\section{Ghaleb Alhakmi, Abderrazzak Assani, Mohamed Saadi and Lahcen El Ammari}

\section{Computing details}

Data collection: APEX2 (Bruker, 2009); cell refinement: SAINT (Bruker, 2009); data reduction: SAINT (Bruker, 2009); program(s) used to solve structure: SHELXS97 (Sheldrick, 2008); program(s) used to refine structure: SHELXL97 (Sheldrick, 2008); molecular graphics: ORTEP-3 for Windows (Farrugia, 2012) and DIAMOND (Brandenburg, 2006); software used to prepare material for publication: publCIF (Westrip, 2010).

Dimanganese(II) zinc bis[orthophosphate(V)] monohydrate

Crystal data

$\mathrm{Mn}_{2} \mathrm{Zn}\left(\mathrm{PO}_{4}\right)_{2} \cdot \mathrm{H}_{2} \mathrm{O}$

$M_{r}=383.21$

Monoclinic, $P 2_{1} / n$

Hall symbol: -P 2yn

$a=8.1784(2) \AA$

$b=10.1741(2) \AA$

$c=9.0896(2) \AA$

$\beta=114.142(1)^{\circ}$

$V=690.17(3) \AA^{3}$

$Z=4$

\section{Data collection}

Bruker X8 APEX

diffractometer

Radiation source: fine-focus sealed tube

Graphite monochromator

$\varphi$ and $\omega$ scans

Absorption correction: multi-scan

(SADABS; Bruker, 2009)

$T_{\min }=0.574, T_{\max }=0.748$

\section{Refinement}

Refinement on $F^{2}$

Least-squares matrix: full

$R\left[F^{2}>2 \sigma\left(F^{2}\right)\right]=0.019$

$w R\left(F^{2}\right)=0.052$

$S=1.11$

2407 reflections

127 parameters

0 restraints

Primary atom site location: structure-invariant direct methods
$F(000)=736$

$D_{\mathrm{x}}=3.688 \mathrm{Mg} \mathrm{m}^{-3}$

Mo $K \alpha$ radiation, $\lambda=0.71073 \AA$

Cell parameters from 2407 reflections

$\theta=2.8-32.0^{\circ}$

$\mu=7.54 \mathrm{~mm}^{-1}$

$T=296 \mathrm{~K}$

Parallelepiped, off-white

$0.32 \times 0.27 \times 0.19 \mathrm{~mm}$

11327 measured reflections

2407 independent reflections

2305 reflections with $I>2 \sigma(I)$

$R_{\text {int }}=0.023$

$\theta_{\text {max }}=32.0^{\circ}, \theta_{\min }=2.8^{\circ}$

$h=-12 \rightarrow 12$

$k=-14 \rightarrow 15$

$l=-13 \rightarrow 13$

Secondary atom site location: difference Fourier map

Hydrogen site location: inferred from neighbouring sites

$\mathrm{H}$-atom parameters constrained

$w=1 /\left[\sigma^{2}\left(F_{\mathrm{o}}^{2}\right)+(0.0248 P)^{2}+1.0141 P\right]$

where $P=\left(F_{\mathrm{o}}^{2}+2 F_{\mathrm{c}}^{2}\right) / 3$

$(\Delta / \sigma)_{\max }=0.001$

$\Delta \rho_{\max }=0.94 \mathrm{e} \AA^{-3}$

$\Delta \rho_{\min }=-0.84$ e $\AA^{-3}$ 


\section{Special details}

Geometry. All e.s.d.'s (except the e.s.d. in the dihedral angle between two l.s. planes) are estimated using the full covariance matrix. The cell e.s.d.'s are taken into account individually in the estimation of e.s.d.'s in distances, angles and torsion angles; correlations between e.s.d.'s in cell parameters are only used when they are defined by crystal symmetry. An approximate (isotropic) treatment of cell e.s.d.'s is used for estimating e.s.d.'s involving 1.s. planes.

Refinement. Refinement of $F^{2}$ against all reflections. The weighted $R$-factor $w R$ and goodness of fit $S$ are based on $F^{2}$, conventional $R$-factors $R$ are based on $F$, with $F$ set to zero for negative $F^{2}$. The threshold expression of $F^{2}>2 \sigma\left(F^{2}\right)$ is used only for calculating $R$-factors $(\mathrm{gt}) e t c$. and is not relevant to the choice of reflections for refinement. $R$-factors based on $F^{2}$ are statistically about twice as large as those based on $F$, and $R$ - factors based on all data will be even larger.

Fractional atomic coordinates and isotropic or equivalent isotropic displacement parameters $\left(\hat{A}^{2}\right)$

\begin{tabular}{lllll}
\hline & $x$ & $y$ & $z$ & $U_{\text {iso }} * / U_{\text {eq }}$ \\
\hline Mn1 & $0.88638(3)$ & $0.35884(3)$ & $0.46580(3)$ & $0.00768(6)$ \\
Mn2 & $0.48057(3)$ & $0.38305(3)$ & $0.21880(3)$ & $0.00726(6)$ \\
Zn1 & $0.12609(3)$ & $0.62028(2)$ & $0.06179(2)$ & $0.00934(6)$ \\
P1 & $0.70438(5)$ & $0.08456(4)$ & $0.32706(5)$ & $0.00553(8)$ \\
P2 & $0.38560(5)$ & $0.67442(4)$ & $0.36388(5)$ & $0.00613(8)$ \\
O1 & $0.58212(17)$ & $0.03301(13)$ & $0.40831(15)$ & $0.0102(2)$ \\
O2 & $0.87050(16)$ & $0.15076(13)$ & $0.45546(15)$ & $0.0094(2)$ \\
O3 & $0.59293(17)$ & $0.18429(12)$ & $0.19887(15)$ & $0.0092(2)$ \\
O4 & $0.76145(17)$ & $-0.03217(12)$ & $0.25178(15)$ & $0.0092(2)$ \\
O5 & $0.23736(18)$ & $0.77279(13)$ & $0.26723(16)$ & $0.0123(2)$ \\
O6 & $0.36400(17)$ & $0.63194(13)$ & $0.51688(15)$ & $0.0104(2)$ \\
O7 & $0.57269(16)$ & $0.73311(13)$ & $0.41028(15)$ & $0.0110(2)$ \\
O8 & $0.35411(17)$ & $0.55914(13)$ & $0.24330(15)$ & $0.0107(2)$ \\
O9 & $0.88135(18)$ & $0.58568(14)$ & $0.57419(16)$ & $0.0130(2)$ \\
H1 & 0.7876 & 0.6203 & 0.4923 & $0.019 *$ \\
H2 & 0.8793 & 0.5969 & 0.6731 & $0.019 *$ \\
\hline
\end{tabular}

Atomic displacement parameters $\left(\AA^{2}\right)$

\begin{tabular}{lllllll}
\hline & $U^{11}$ & $U^{22}$ & $U^{33}$ & $U^{12}$ & $U^{13}$ & $U^{23}$ \\
\hline Mn1 & $0.00519(11)$ & $0.00776(11)$ & $0.00860(11)$ & $-0.00070(8)$ & $0.00129(8)$ & $0.00274(8)$ \\
Mn2 & $0.00657(11)$ & $0.00704(11)$ & $0.00777(11)$ & $-0.00016(8)$ & $0.00254(9)$ & $-0.00025(8)$ \\
Zn1 & $0.00839(10)$ & $0.00949(10)$ & $0.00884(9)$ & $0.00028(6)$ & $0.00219(7)$ & $0.00093(6)$ \\
P1 & $0.00538(16)$ & $0.00594(17)$ & $0.00519(16)$ & $0.00046(13)$ & $0.00209(13)$ & $-0.00030(13)$ \\
P2 & $0.00556(16)$ & $0.00676(17)$ & $0.00569(16)$ & $0.00008(13)$ & $0.00193(13)$ & $-0.00044(13)$ \\
O1 & $0.0107(5)$ & $0.0111(5)$ & $0.0126(5)$ & $0.0015(4)$ & $0.0087(5)$ & $0.0025(4)$ \\
O2 & $0.0079(5)$ & $0.0105(5)$ & $0.0073(5)$ & $-0.0020(4)$ & $0.0008(4)$ & $-0.0025(4)$ \\
O3 & $0.0099(5)$ & $0.0080(5)$ & $0.0081(5)$ & $0.0022(4)$ & $0.0020(4)$ & $0.0013(4)$ \\
O4 & $0.0083(5)$ & $0.0091(5)$ & $0.0099(5)$ & $0.0017(4)$ & $0.0033(4)$ & $-0.0027(4)$ \\
O5 & $0.0121(5)$ & $0.0135(6)$ & $0.0108(5)$ & $0.0070(5)$ & $0.0041(4)$ & $0.0035(4)$ \\
O6 & $0.0106(5)$ & $0.0137(6)$ & $0.0071(5)$ & $-0.0006(4)$ & $0.0038(4)$ & $0.0016(4)$ \\
O7 & $0.0081(5)$ & $0.0129(6)$ & $0.0120(5)$ & $-0.0031(4)$ & $0.0041(4)$ & $-0.0030(4)$ \\
O8 & $0.0107(5)$ & $0.0093(5)$ & $0.0099(5)$ & $0.0011(4)$ & $0.0021(4)$ & $-0.0035(4)$ \\
O9 & $0.0109(5)$ & $0.0181(6)$ & $0.0106(5)$ & $0.0020(5)$ & $0.0051(4)$ & $0.0007(5)$ \\
& & & & & &
\end{tabular}


Geometric parameters $\left(\AA,{ }^{\circ}\right)$

\begin{tabular}{|c|c|c|c|}
\hline $\mathrm{Mn} 1-\mathrm{O}^{\mathrm{i}}$ & $2.1191(13)$ & $\mathrm{Zn} 1-\mathrm{O}^{\mathrm{vi}}$ & $2.0242(13)$ \\
\hline $\mathrm{Mn} 1-\mathrm{O} 2$ & $2.1208(14)$ & $\mathrm{Zn} 1-\mathrm{O} 1^{\mathrm{viii}}$ & $2.0347(12)$ \\
\hline $\mathrm{Mn} 1-\mathrm{O}^{\mathrm{ii}}$ & $2.1464(12)$ & $\mathrm{Zn} 1-\mathrm{O} 5$ & $2.3093(14)$ \\
\hline $\mathrm{Mn} 1-\mathrm{O} 9^{\mathrm{iii}}$ & $2.1504(14)$ & $\mathrm{P} 1-\mathrm{O} 3$ & $1.5327(13)$ \\
\hline $\mathrm{Mn} 1-\mathrm{O} 4^{\mathrm{iv}}$ & $2.1556(13)$ & $\mathrm{P} 1-\mathrm{O} 4$ & $1.5355(13)$ \\
\hline $\mathrm{Mn} 1-\mathrm{O} 9$ & $2.5163(15)$ & $\mathrm{P} 1-\mathrm{O} 2$ & $1.5377(13)$ \\
\hline $\mathrm{Mn} 2-\mathrm{O} 8$ & $2.1254(13)$ & $\mathrm{P} 1-\mathrm{O} 1$ & $1.5570(13)$ \\
\hline $\mathrm{Mn} 2-\mathrm{O}^{\mathrm{v}}$ & $2.1533(13)$ & $\mathrm{P} 2-\mathrm{O} 7$ & $1.5322(13)$ \\
\hline $\mathrm{Mn} 2-\mathrm{O} 4^{\mathrm{iv}}$ & $2.1921(13)$ & $\mathrm{P} 2-\mathrm{O} 6$ & $1.5340(13)$ \\
\hline $\mathrm{Mn} 2-\mathrm{O} 2^{\mathrm{vi}}$ & $2.2126(13)$ & $\mathrm{P} 2-\mathrm{O} 5$ & $1.5401(13)$ \\
\hline $\mathrm{Mn} 2-\mathrm{O}^{\mathrm{i}}$ & $2.2166(13)$ & $\mathrm{P} 2-\mathrm{O} 8$ & $1.5532(13)$ \\
\hline $\mathrm{Mn} 2-\mathrm{O} 3$ & $2.2590(13)$ & $\mathrm{O} 9-\mathrm{H} 1$ & 0.8939 \\
\hline $\mathrm{Zn} 1-\mathrm{O} 7^{\mathrm{vii}}$ & $1.9546(13)$ & $\mathrm{O} 9-\mathrm{H} 2$ & 0.9131 \\
\hline $\mathrm{Zn} 1-\mathrm{O} 8$ & $2.0174(13)$ & & \\
\hline $\mathrm{O} 6-\mathrm{Mn} 1-\mathrm{O} 2$ & $90.23(5)$ & $\mathrm{O} 4^{\mathrm{iv}}-\mathrm{Mn} 2-\mathrm{O} 3$ & $87.66(5)$ \\
\hline $\mathrm{O} 6^{\mathrm{i}}-\mathrm{Mn} 1-\mathrm{O} 3^{\mathrm{ii}}$ & $109.27(5)$ & 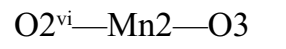 & $76.87(5)$ \\
\hline $\mathrm{O} 2-\mathrm{Mn} 1-\mathrm{O} 3^{\mathrm{ii}}$ & $81.31(5)$ & $\mathrm{O} 6-\mathrm{Mn} 2-\mathrm{O} 3$ & $87.34(5)$ \\
\hline $\mathrm{O} 6^{\mathrm{i}}-\mathrm{Mn} 1-\mathrm{O} 9^{\mathrm{iii}}$ & $161.48(5)$ & 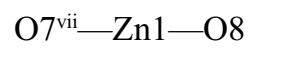 & $132.60(5)$ \\
\hline $\mathrm{O} 2-\mathrm{Mn} 1-\mathrm{O} 9^{\mathrm{iii}}$ & $107.27(5)$ & $\mathrm{O} 7^{\mathrm{vii}}-\mathrm{Zn} 1-\mathrm{O} 1^{\mathrm{vi}}$ & $100.19(6)$ \\
\hline 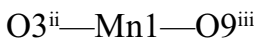 & $80.06(5)$ & $\mathrm{O} 8-\mathrm{Zn} 1-\mathrm{O}^{\mathrm{vi}}$ & $99.81(5)$ \\
\hline $\mathrm{O} 6^{\mathrm{i}}-\mathrm{Mn} 1-\mathrm{O} 4^{\mathrm{iv}}$ & $81.32(5)$ & $\mathrm{O} 7^{\mathrm{vii}}-\mathrm{Zn} 1-\mathrm{O} 1^{\mathrm{viii}}$ & $117.98(5)$ \\
\hline $\mathrm{O} 2-\mathrm{Mn} 1-\mathrm{O} 4^{\mathrm{iv}}$ & $118.14(5)$ & $\mathrm{O} 8-\mathrm{Zn} 1-\mathrm{O} 1^{\mathrm{viii}}$ & $107.48(5)$ \\
\hline 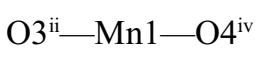 & $158.49(5)$ & 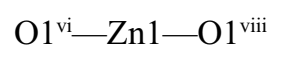 & $80.41(5)$ \\
\hline $\mathrm{O} 9^{\mathrm{iii}}-\mathrm{Mn} 1-\mathrm{O} 4^{\mathrm{iv}}$ & $84.99(5)$ & $\mathrm{O} 7^{\mathrm{vii}-\mathrm{Zn} 1-\mathrm{O} 5}$ & $87.57(5)$ \\
\hline O6 $-\mathrm{Mn} 1-\mathrm{O} 9$ & $76.07(5)$ & $\mathrm{O} 8-\mathrm{Zn} 1-\mathrm{O} 5$ & $67.61(5)$ \\
\hline $\mathrm{O} 2-\mathrm{Mn} 1-\mathrm{O} 9$ & $157.28(5)$ & $\mathrm{O} 1^{\mathrm{vi}-\mathrm{Zn} 1-\mathrm{O} 5}$ & $167.20(5)$ \\
\hline $\mathrm{O} 3^{\mathrm{ii}}-\mathrm{Mn} 1-\mathrm{O} 9$ & $86.18(5)$ & $\mathrm{O} 1^{\text {viii-Zn1-O5 }}$ & $105.05(5)$ \\
\hline O9ii-Mn1-O9 & $89.00(5)$ & $\mathrm{O} 3-\mathrm{P} 1-\mathrm{O} 4$ & $111.58(7)$ \\
\hline $\mathrm{O} 4^{\mathrm{iv}}-\mathrm{Mn} 1-\mathrm{O} 9$ & $78.15(5)$ & $\mathrm{O} 3-\mathrm{P} 1-\mathrm{O} 2$ & $110.68(7)$ \\
\hline $\mathrm{O} 8-\mathrm{Mn} 2-\mathrm{O}^{\mathrm{v}}$ & $89.03(5)$ & $\mathrm{O} 4-\mathrm{P} 1-\mathrm{O} 2$ & $109.99(7)$ \\
\hline $\mathrm{O} 8-\mathrm{Mn} 2-\mathrm{O} 4^{\text {iv }}$ & $98.10(5)$ & $\mathrm{O} 3-\mathrm{P} 1-\mathrm{O} 1$ & $106.62(7)$ \\
\hline $\mathrm{O}^{\mathrm{v}}-\mathrm{Mn} 2-\mathrm{O}_{4}^{\mathrm{iv}}$ & $167.53(5)$ & $\mathrm{O} 4-\mathrm{P} 1-\mathrm{O} 1$ & $108.79(7)$ \\
\hline $\mathrm{O} 8-\mathrm{Mn} 2-\mathrm{O} 2^{\mathrm{vi}}$ & $104.14(5)$ & $\mathrm{O} 2-\mathrm{P} 1-\mathrm{O} 1$ & $109.08(7)$ \\
\hline $\mathrm{O} 5^{\mathrm{v}}-\mathrm{Mn} 2-\mathrm{O} 2^{\mathrm{vi}}$ & $90.14(5)$ & $\mathrm{O} 7-\mathrm{P} 2-\mathrm{O} 6$ & $109.42(7)$ \\
\hline $\mathrm{O} 4^{\mathrm{iv}}-\mathrm{Mn} 2-\mathrm{O} 2^{\mathrm{vi}}$ & $97.96(5)$ & $\mathrm{O} 7-\mathrm{P} 2-\mathrm{O} 5$ & $111.62(8)$ \\
\hline $\mathrm{O} 8-\mathrm{Mn} 2-\mathrm{O}^{\mathrm{i}}$ & $91.85(5)$ & $\mathrm{O} 6-\mathrm{P} 2-\mathrm{O} 5$ & $110.15(7)$ \\
\hline $\mathrm{O}^{\mathrm{v}}-\mathrm{Mn} 2-\mathrm{O} 6^{\mathrm{i}}$ & $91.25(5)$ & $\mathrm{O} 7-\mathrm{P} 2-\mathrm{O} 8$ & $110.32(7)$ \\
\hline $\mathrm{O} 4^{\mathrm{iv}}-\mathrm{Mn} 2-\mathrm{O}^{\mathrm{i}}$ & $78.36(5)$ & $\mathrm{O} 6-\mathrm{P} 2-\mathrm{O} 8$ & $112.31(8)$ \\
\hline $\mathrm{O} 2^{\mathrm{vi}}-\mathrm{Mn} 2-\mathrm{O}^{\mathrm{i}}$ & $163.97(5)$ & $\mathrm{O} 5-\mathrm{P} 2-\mathrm{O} 8$ & $102.92(7)$ \\
\hline $\mathrm{O} 8-\mathrm{Mn} 2-\mathrm{O} 3$ & $173.90(5)$ & $\mathrm{H} 1-\mathrm{O} 9-\mathrm{H} 2$ & 114.6 \\
\hline $\mathrm{O}^{\mathrm{v}}-\mathrm{Mn} 2-\mathrm{O} 3$ & $84.95(5)$ & & \\
\hline
\end{tabular}

Symmetry codes: (i) $-x+1,-y+1,-z+1$; (ii) $x+1 / 2,-y+1 / 2, z+1 / 2$; (iii) $-x+2,-y+1,-z+1$; (iv) $-x+3 / 2, y+1 / 2,-z+1 / 2$; (v) $-x+1 / 2, y-1 / 2,-z+1 / 2$; (vi) $x-1 / 2,-y+1 / 2, z-1 / 2 ;$ (vii) $x-1 / 2,-y+3 / 2, z-1 / 2$; (viii) $-x+1 / 2, y+1 / 2,-z+1 / 2$. 


\section{supporting information}

Hydrogen-bond geometry $\left(\AA,{ }^{\circ}\right)$

\begin{tabular}{lllll}
\hline$D-\mathrm{H} \cdots A$ & $D-\mathrm{H}$ & $\mathrm{H} \cdots A$ & $D \cdots A$ & $D-\mathrm{H} \cdots A$ \\
\hline $\mathrm{O} 9-\mathrm{H} 1 \cdots \mathrm{O} 7$ & 0.89 & 1.97 & $2.7866(19)$ & 151 \\
$\mathrm{O}^{-}-\mathrm{H} 2 \cdots{ }^{\text {ix }}$ & 0.91 & 2.16 & $2.8687(19)$ & 134 \\
$\mathrm{O}^{-}-\mathrm{H} 2 \cdots{ }^{\mathrm{ii}}$ & 0.91 & 2.48 & $3.0494(19)$ & 120
\end{tabular}

Symmetry codes: (ii) $x+1 / 2,-y+1 / 2, z+1 / 2$; (ix) $x+1 / 2,-y+3 / 2, z+1 / 2$. 